\title{
Psychological distress among patients with organophosphorus or paraquat poisoning
}

\author{
Jayasinghe $\mathrm{SS}^{1,2}$ \\ ${ }^{\prime}$ Department of Pharmacology, Faculty of Medicine, University of Ruhuna, Sri Lanka \\ ${ }^{2}$ South Asian Clinical Toxicology Research Collaboration, Department of Medicine, Faculty of Medicine, \\ University of Peradeniya, Sri Lanka
}

Correspondence: Dr.S.S.Jayasinghe (sudheerasj@yahoo.com)

\begin{abstract}
Introduction: Recognition of psychological distress following intentional self poisoning is important. The most widely used screening test for psychological distress is the Goldberg's General Health Questionnaire (GHQ). It is available in versions as short as 12 items (GHQ-12) and as long as 60 (GHQ-60).

Objective: The aim of the study was to determine the prevalence of psychological distress among patients with self poisoning with organophosphorus (OP) or paraquat (PQ) pesticides admitted to the General Hospital, Matara and the Teaching Hospital, Galle.

Method: A case control study was conducted with matched controls. A self-administered validated Sinhala translation of the 12-item Goldberg's General Health Questionnaire (GHQ-12) was used as a general screening test to identify psychological distress. A score $>15$ indicates evidence of psychological distress and a score $>20$ is suggestive of severe psychological problems and distress. A score $>15$ is also considered as positive GHQ-12.

Results: GHQ-12 was administered to 143 (99 OP, 44 PQ) patients and 70 controls. Mean (SD) ages of the test and control group were 34 (14) and 33 (12) is respectively. The prevalence of psychological distress among the patients and the controls were 51\% (73/143) and 10\% (7/70), respectively. The median (inter-quartile range) of GHQ-12 scores among patients and controls were 16(11 23) and 8 (5 11), respectively $(\mathrm{P}<0.001)$.

Conclusions: This study indicates the high prevalence of psychological distress among patients with self poisoning with OP or PQ and emphasizes the need of assessment, support and if required treatment in needy cases..
\end{abstract}

Keywords: Organophosphorus compounds, Paraquat, Suicide

\section{Introduction}

Deliberate self harm is a common health problem in developing countries. Suicide rates in Sri Lanka increased by $700 \%$ between 1960 to 1997 and this was entirely due to an increase in deaths by poisoning (1). In Sri Lanka pesticide poisoning has ranked as the fifth or sixth leading cause of death (2). Among substances used for suicide, organophosphorus pesticides (OP) are the commonly used. Although paraquat (PQ) poisoning is less common it is a major health problem due to its high case fatality $(3,4)$. It is a common belief that psychiatric illness and suicidal attempts are related.
However literature does not support a significant relationship between self-poisoning and psychological distress. Psychological status even after an attempted suicide is not always addressed (5) and some individuals have multiple attempts of suicide. Therefore identification and management of psychological distress following suicidal attempts are important to minimize future suicide risk. The aim of this study was to determine the prevalence of psychological distress among patients with selfpoisoning of OP or PQ, admitted to the General Hospital, Matara and the Teaching Hospital, Galle. 


\section{Methods}

A case control study was conducted with controls matched for age, gender and level of education. Ethical approval for the study was granted by the Ethical Review Committee, Faculty of Medicine, University of Ruhuna. Participants were recruited from the General Hospital, Matara (from June 2008 July 2009) and the Teaching Hospital, Galle (from August 2008 December 2009). Consecutive patients admitted with self ingestion of OP or PQ and matched controls were enrolled in the study after obtaining written informed consent.

The controls were recruited from persons accompanying patients to medical (other than poisoning) or surgical wards of the Teaching Hospital, Galle. Detailed history was taken to make sure that they did not have a history of acute pesticide exposure. The controls were matched to \pm 3 years of the age of cases. Gender and the category of educational level were matched too (Table 1).
The General Health Questionnaire (GHQ) is the most widely used screening test in detecting short term psychological distress $(6,7)$. It can be used for medical outpatients or inpatients (6). GHQ is available as short as 12 items and as long as 60 items (6).

The Sinhala version of the 12-items GHQ (GHQ-12) was used in the current study. GHQ-12 is a self administered questionnaire with 12 questions. Each question has four responses. GHQ-12 items are rated using a Likert-type scale from 0-3 with a range of 036. A GHQ-12 score of more than 15 is suggestive of distress and a total score more than 20 suggests severe psychological distress $(8,9)$. The questionnaire was administered once the patient had recovered from the acute clinical problem and at the time of discharge from the hospital.

Statistical analysis was performed using GraphPad prism 4 and Epi info. Unpaired t-test was used to compare the results of the patients and the controls. Potential confounders were adjusted with multiple linear regressions.

Table 1: Descriptive data of the participants

\begin{tabular}{lll}
\hline Descriptive data & Patients (N=143) & Controls (N=70) \\
\hline Age (years) & $34 \pm 14$ & $33 \pm 12$ \\
Gender (Male) & $108(76 \%)$ & $51(73 \%)$ \\
Marital status (married) & $96(68 \%)$ & $48(69 \%)$ \\
Educational level & & \\
$\quad$ Never gone to school & $09(6 \%)$ & $03(4 \%)$ \\
$\quad$ Grade 1-5 & $24(17 \%)$ & $06(9 \%)$ \\
$\quad$ Grade 6-10 & $67(48 \%)$ & $38(54 \%)$ \\
$\quad$ Grade 11-12 & $40(28 \%)$ & $20(29 \%)$ \\
$\quad$ Higher education & $01(0.7 \%)$ & $03(4 \%)$ \\
Smoking habits & & \\
$\quad$ Never smoke & $74(53 \%)$ & $43(61 \%)$ \\
$\quad$ Occasional & $09(6 \%)$ & $25(25 \%)$ \\
$\quad$ Regular & $58(41 \%)$ & $02(3 \%)$ \\
Alcohol consumption & & \\
$\quad$ Never & $67(48 \%)$ & $43(61 \%)$ \\
$\quad$ Occasional & $39(27 \%)$ & $25(36 \%)$ \\
$\quad$ Regular & $35(25 \%)$ & $02(3 \%)$ \\
Co-morbid disease (yes) & $12(8.5 \%)$ & $0(0 \%)$ \\
GHQ score & & \\
GHQ-12 ? 15 (normal) & $70(49 \%)$ & $63(90 \%)$ \\
GHQ-12=16 to 20 (distress) & $21(15 \%)$ & $06(9 \%)$ \\
GHQ-12? 21 (severe distress) & $52(36 \%)$ & $01(1.5 \%)$ \\
Previous suicidal attempts (yes) & $15(10 \%)$ & $0(0 \%)$ \\
Family history of suicidal attempts (yes) & $16(11 \%)$ & $7(10 \%)$ \\
\hline
\end{tabular}




\section{Results}

A total of 225 patients were screened for entry in to the study. Fifty nine patients died in the hospital and four patients were transferred for intensive care. Fifteen patients were not able to read. Four questionnaires were incompletely filled and excluded from the analysis. Hence questionnaires of 143 (99 OP, 44 PQ) patients were considered for the final analysis. The number of controls was 70 .

The descriptive data, psychological status according to the GHQ-12, previous suicidal attempts and family history of suicidal attempts are shown in Table 1. In two patients the data of marital status, level of education, smoking habits, alcohol consumption and co-morbid factors were not available.

The median (inter-quartile range) of GHQ-12 score in the test and the control groups were 16 (11 23) and 8 (5 11), respectively $(\mathrm{P}<0.001)$ by Mann-Whitney $\mathrm{U}$ test. The prevalence of psychological distress among the test and the control groups were 51\% (73/143) and 10\% (7/70), respectively.

From the 15 patients who had previous suicidal attempts in the test group, three patients had four suicidal attempts and one patient had three suicidal attempts including the current attempt. None of the controls had suicidal attempts. Among the patients who had existing diseases (co-morbid disease) one patient was known to have a psychiatric disorder.
When age, gender, marital status, educational level, co-morbid disease, family history of suicidal attempts, previous suicidal attempts, smoking habits and alcohol consumption were included in the multiple linear regression model, educational level and co-morbid diseases were found to be statistically significant (Table 2).

The prevalence of psychological distress was not significantly different when cases were analyzed based on gender, age or marital status (data not shown)

\section{Discussion}

A high prevalence of psychological distress was found among cases compared to the controls and the level of education and co-morbidity were found to be significant determinants. Furthermore, there was no difference in the prevalence of psychological distress between young and old, male and female or married and single.

The performance of GHQ-12 was shown to be equal to longer versions of GHQs $(10,11)$. The GHQ-12 is a brief self report of measure and has excellent psychometric properties as a screening instrument for psychiatric disorders, and takes only about two minutes to complete $(6,12)$. The GHQ-12 had sensitivity of $83 \%$ and specificity of $79 \%$ with positive predictive value of $64 \%$ (6). The GHQ was validated in Sinhala by Rodrigo EK (13) and it has been tested in Sri Lanka (14).

Table 2: Adjusted multiple linear regression for confounders

\begin{tabular}{llll}
\hline Covariates & $\begin{array}{c}\text { Standardized } \\
\text { Coefficients }\end{array}$ & P value & $\begin{array}{c}\text { 95\% } \\
\text { Confidence }\end{array}$ \\
\hline Age & 0.13 & 0.17 & -0.04 to 0.2 \\
Gender & 0.05 & 0.62 & -3 to 5 \\
Marital status & -0.02 & 0.87 & -4 to 3 \\
Educational level & -0.21 & 0.02 & -4 to -0.3 \\
Co morbid diseases & 0.19 & 0.03 & 0.5 to 11 \\
Previous suicidal attempts & 0.12 & 0.16 & -1 to 8 \\
Family history of suicidal attempts & 0.02 & 0.85 & -5 to 6 \\
Smoking & 0.08 & 0.48 & -1 to 3 \\
Alcohol consumption & -0.11 & 0.31 & -4 to 1 \\
\hline
\end{tabular}


In the current study, $108(76 \%)$ patents were males. It has been shown that females more often attempt suicide, but males more often employ lethal means to do so. Therefore males more often die by suicide than females (15). PQ poisoning has a very high mortality. A case fatality rate over $10 \%$ has been shown in most hospitals following OP ingestion (16). The lethal effect of PQ and OP are thus well known. This may be the reason why the current study consisted of more males.

Joe $\mathrm{S}$ et al (2008) showed that the risk of attempted suicide was significantly associated with being female, being in the age group 18-34 years, having low / medium educational levels, alcohol consumption, use of illicit drugs, symptoms indicative of conduct disorders and depressive symptoms $(17,19)$. The strongest predictor of suicide was a prior experience of non fatal suicide behavior (20). The above factors, co-morbid disease, family history of suicide attempts and smoking habits were identified as possible confounders for GHQ-12 and analyzed with multiple linear regression. The model showed significant association with educational level and co morbid disease only.

The median ages of onset of initial suicide ideation and planning are in the late twenties but for attempts the median age of onset is early thirty (17). In younger age groups unplanned suicide attempts are more common than planned attempts (17). The median age of the current test group was 30 years which is comparable to previous findings.

The high GHQ-12 score and prevalence of psychological distress in the test group indicates that these patients require special attention since suicidal behavior may be a salient sign of a high risk of suicide. Suicidal ideation is the least common symptom of major depressive disorder (21). Psychiatric disorders have been considered to be major causative factors for suicidal behavior and their care should include reducing suicidal behavior recurrence and preventing suicide $(22,23,24)$. Adolescents who report suicidal ideation have a higher probability of axis 1 disorder: problem behaviors and poor coping abilities, low self-esteem levels and interpersonal relations (24).

Mental health services are very important for individuals with suicidal ideation and suicide attempts $(5,25)$. It may not be possible to assess the psychological status of each and every patient with suicidal attempts by a psychiatrist, but it would be possible with a trained mental health worker dedicated for welfare of these patients. Expansion of psychological services with counseling and identification of patients who need further psychological support and management is important. The prevalence of self perceived need for mental health care among those with suicidal behaviors is unclear, but those who do not perceive a need are unlikely to use services $(5,25)$.

\section{Limitations}

Only two groups of people with suicidal behavior (those who ingested PQ or OP) were selected for the study. It did not represent the entire population who attempted suicide and these findings are not generalizable. GHQ-12, which is the shortest version of GHQ was used for the current study. The longer versions may give additional information. The GHQ-12 was administered during the hospital stay of the patients and unfamiliar hospital environment and staff may have contributed to their psychological status at the time of testing.

\section{Acknowledgements}

We would like to thank the participants, the consultants who gave patients for the study, administrative staff and health care professionals at the Teaching Hospital, Galle and the General Hospital, Matara, Heads and the staff members of the Department of Pharmacology and the Department of Medicine, Faculty of Medicine, University of Ruhuna, and the members and the Clinical Research Assistances of the South Asian Clinical Toxicology Research Collaboration. A special word of thanks is extended to Professor NA Buckley, Professor AH Dawson, Professor A Fernando, Professor KD Pathirana, Professor PL Ariyananda and Dr Bilesha Perera.

\section{Source of funding}

This study was supported by a Wellcome Trust and NHMRC International Collaborative Research Grant (GR071669MA). 


\section{References}

1. Van der Hoek W, Konradsen F. Risk factors for acute pesticide poisoning in Sri Lanka. Trop Med Int Health, 2005; 10(6): 589-96.

2. Annual Health Bulletin. Morbidity and mortality. In: Health Ministry Sri Lanka; 2003: 21-30.

3. Wilks MF, Fernando R, Ariyananda PL, Eddleston M, Berry DJ, Tomenson JA, et al. Improvement in survival after paraquat ingestion following introduction of a new formulation in Sri Lanka. PLoS Med, 2008; 5(2): e49.

4. Gawarammana IB, Dawson AH. Peripheral burning sensation: a novel clinical marker of poor prognosis and higher plasma-paraquat concentrations in paraquat poisoning. Clin Toxicol (Phila), 2010; 48(4): 347-9.

5. Pagura J, Fotti S, Katz L Y, Sareen J. Help seeking and perceived need for mental health care among individuals in Canada with suicidal behaviors. Psychiatr Serv, 2009; 60(7): 943-9.

6. Goldberg D. Identifying psychiatric illness among general patients. BMJ, 1985:291.

7. Abeysena C, Jayawardana P, Seneviratne RA. Effect of psychological stress on maternal complications during pregnancy: A cohort study. International Journal of Collaborative Research on Internal Medicine \& Public Health, 2010; 2(12): 436-48.

8. Gao F, Luo N, Thumboo J, Fones C, Li SC, Cheung YB. Does the 12-item General Health Questionnaire contain multiple factors and do we need them? Health Qual Life Outcomes, 2004; 2: 63.

9. Schmitz N, Kruse J, Tress W. Psychometric properties of the General Health Questionnaire (GHQ-12) in a German primary care sample. Acta Psychiatr Scand, 1999; 100(6): $462-8$.

10. Kearns NP, Cruickshank CA, McGuigan KJ, Riley SA, Shaw S, Snaith R. A comparison of depression rating scales. BrJPsychiatry, 1982; 141: 45-9.

11. Zigmond AS, Snaith R. The hospital anxiety and depression scale. Acta Psychiatr Scand, 1983; 67(6): 361-70.

12. Hu Y, Stewart-Brown S, Twigg L, Weich S. Can the 12-item General Health Questionnaire be used to measure positive mental health? Psychol Med, 2007; 37(7): 1005-13.

13. Kuruppuarachchi KALA, Kuruppuarachchi KAJM, Wijerathne S, Williams SS. Psychological distress among students from five universities in Sri Lanka. Ceylon Medical Journal, 2002; 47(1): 104-105.
14. Chakrabhan ML, Chandra V, Levav I, Pengjuntr W, Bhugra D, Mendis N, et al. Panel 2.6: mental and psychosocial effects of the Tsunami on the affected populations. Prehosp Disaster Med, 2005; 20(6): 414-9.

15. Kerr DC, Owen LD, Pears KC, Capaldi DM. Prevalence of suicidal ideation among boys and men assessed annually from ages 9 to 29 years. Suicide Life Threat Behav, 2008; 38(4): 390-402.

16. Buckley NA, Eddleston M, Andrew H Dawson. The need for translational research on antidotes for pesticide poisoning. Clin Exp Pharmacol Physiol 2005; 32(11): 9991005.

17. Joe S, Stein DJ, Seedat S, Herman A, Williams DR. Nonfatal suicidal behavior among South Africans: results from the South Africa Stress and Health Study. Soc Psychiatry Psychiatr Epidemiol, 2008; 43(6): 454-61.

18. Joe S, Stein D J, Seedat S, Herman A, Williams DR. Prevalence and correlates of non-fatal suicidal behaviour among South Africans. Br J Psychiatry, 2008; 192(4): 3101.

19. Souza LD, Silva R, Jansen K, Kuhn RP, Horta BL, Pinheiro RT. Suicidal ideation in adolescents aged 11 to 15 years: prevalence and associated factors. Rev Bras Psiquiatr, 2010; 32(1): 37-41.

20. Harris EC, Barraclough B. Suicide as an outcome for mental disorders. A meta-analysis. Br J Psychiatry, 1997; 170: 205-28.

21. Roberts RE, Lewinsohn PM, Seeley JR. Symptoms of DSM-III-R major depression in adolescence: evidence from an epidemiological survey. J Am Acad Child Adolesc Psychiatry, 1995;34(12): 1608-17.

22. Cavanagh JT, Carson AJ, Sharpe M, Lawrie SM. Psychological autopsy studies of suicide: a systematic review. Psychol Med, 2003; 33(3): 395-405.

23. Work group of suicidal behaviours of the American Psychiatric Association. Practice guideline for the assessment and treatment of patients with suicidal behaviors. Am J Psychiatry, 2003; 160(11 Suppl): 1-60.

24. Reinherz HZ, Tanner JL, Berger SR, Beardslee WR, Fitzmaurice GM. Adolescent suicidal ideation as predictive of psychopathology, suicidal behavior, and compromised functioning at age 30. Am J Psychiatry, 2006; 163(7): 122632.

25. Edlund M, Unutzer J, Curran GM. Perceived need for alcohol, drug, and mental health treatment. Soc Psychiatry Psychiatr Epidemiol, 2006; 41(6): 480-7. 